\title{
CORRELATION? WHAT CORRELATION?
}

\author{
J.H. EBERLY \\ Department of Physics and Astronomy, University of Rochester \\ Rochester NY 14627, USA
}

\begin{abstract}
We describe recent developments in the theory of multi-electron atoms in strong radiation ficlds, with particular focus on the degree of e-e correlation accompanying a laser-atom interaction.
\end{abstract}

PACS numbers: $32.80 . \mathrm{Fb}, 32.80 . \mathrm{Rm}, 42.50 . \mathrm{IIz}$

In a series of papers [1-5] we have explored the response of a one-electron atom to intense short-pulse laser fields in a reduced-dimension atom model. This 1D "atom" appears to be reasonably realistic, at least in the presence of powerful linearly polarized laser light that induces the single electron to oscillate along the polarization axis. The binding potential for this model is (in atomic units)

$$
V(x)=-1 / \sqrt{x^{2}+1}
$$

Our atom can be said to represent hydrogen, but the lack of singularity of $V(x)$ near to $x=0$ makes it more like an alkali atom with a single electron outside a large frozen core. In any event, the simplicity of a single space dimension makes the integration of Schrödinger's time-dependent wave equation numerically feasible over a very large spatial grid, even for laser fields so strong that perturbation theory is unreliable. Using the numerically exact time-dependent field-dependent wave functions obtained in this way for this atom, dynamical features of above-threshold ionization, high-order harmonic production and atomic stabilization have been studied in close detail [1-5].

More recently we have begun to use the same potential in the two-electron case. A solution of the two-electron Schrödinger equation is significantly more challenging of computer resources, and it is not yet feasible in three space dimensions without basis-set restrictions. However, some progress can be made one-dimensionally, and preliminary results have been reported [6-9]. If we label the two electron coordinates by $x$ and $y$, the natural two-electron potential is

$$
V(x, y)=-Z V(x)-Z V(y)+V(x-y),
$$

where $V$ is as given in (1), and $Z$ is the nuclear charge. Of course the important new feature here is the electron-electron repulsion term. We point out that in this model the e-e repulsion obeys the same potential as the electron-nuclear attraction, as in real atom.

The first task is to understand the structure of such an atom, so we have solved the time-independent Schrödinger equation to find eigenvalues and wave 
functions for the lowest bound states as well as appropriate continuum states. This is done directly numerically [9] on the $x-y$ spatial grid without basis state expansions or assumptions. To do this systematically we first discard the e-e repulsion and find the energies for the fully decorrelated two-electron atom. Then the repulsion term is added back, but multiplied by a small numerical parameter $G$. For very small $G$ the exact energies deviate only little from their uncorrelated values, and they can be checked against perturbation theory, so their labels (quantum number assignments) are not ambiguous. A small increase in $G$ gives a new set of energies, easily connected to the previous ones, and so on until the desired physical value $G=1$ is reached. Figure 1 shows this progression of energies as a

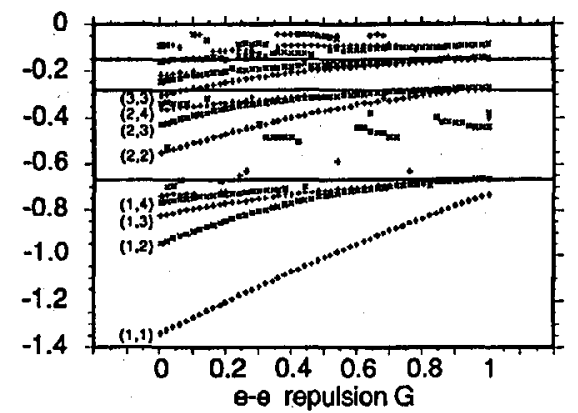

Fig. 1. The total two-electron energies for the potential $V(x, y)$ given in Eq. (2), when $Z=1$ and the e-e repulsion term is multiplied by the factor $G$, plotted as a function of $G$. At each value of $G$ a numerical energy spectrum is obtained, and the numerically determined positions of spectral maxima are indicated by + and $\times$ signs. The main energy level sequences are easy to identify. Occasional false peaks that arise from minor numerical fluctuations also occur.

function of $G$ for the negative ion case, when $Z=1$. This is the most interesting case because the $\mathrm{e}-\mathrm{e}$ correlation is the strongest. The most prominent identifying feature of negative ions in the real world of $3 \mathrm{D}$ atoms is their single bound energy level, and we see from Fig. 1 that our 1D model shares this feature: by the time the threshold value $G=1$ is reached, all but one of the bound energies have passed into the continuum.

The existence of a strong e-e correlation in the model makes it an ideal "laboratory" in which to test the effect of correlation during an intense and short laser pulse. The interplay of the two electrons during laser excitation is shown in Figs. 2 and 3. Figure 2a shows the detachment probability of the "outer" electron as a function of time for three different electric field strengths (in a.u.), and it is clear that as the electric field strength is increased the detachment process occurs more and more rapidly. For $\mathcal{E}=0.01$ a.u. the electron is completely detached (probability equals 1 ) by $t=10$ optical cycles. However, note that Fig. 2b shows a decreased detachment probability for an electric field strength still larger by a factor of 2. This reversal of form, i.e., the decreased ionization with increased field strength, is one indication of atomic stabilization, calculated by us for the first 

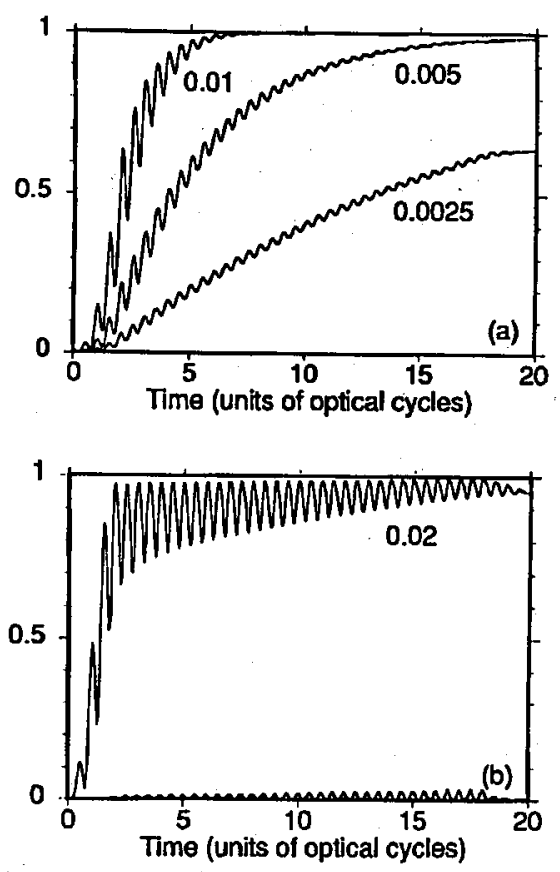

Fig. 2. The detachment probability as a function of time labelled by values of the electric field strength. (a) Increases in field strength lead to more and more prompt detachment. (b) A further increase in field strength leads to a decrease in detachment probability. The lower curve shows that in this case the field strength happens to be high enough to excite a small probability of core ionization as well as detachment.

time for a two-electron system [7]. Figure 3 shows some of the same data in its spatial representation. The two spatial probability distributions correspond to the temporal probabilities of Fig. 2 at the time $t=13$ cycles for the two highest field strengths shown, namcly $\mathcal{E}=0.01$ and 0.02 a.u.

Another example of e-e correlation first reported by Grobe and Eberly [8] can be termed coherence transfer by e-e correlation. In Fig. 4 there is a two-electron energy level diagram. The right side shows the single discrete state and the one electron thresholds above it, while the left side pretends that energies can be labelled as belonging to "outer" and "core" electrons (ignoring the fact that our wave functions are properly symmetrized), in order to represent more pictorially the phenomenon of interest, in which the outer electron is being photodetached while the core undergoes resonant excitation. The photodetachment energy spectrum is shown split into two peaks, which we have called a coherence transfer doublet. A core resonance was predicted by Knight to lead to a split photo-spectrum many years ago [10], but the "Knight doublet" is a one-electron effect. In Fig. 5 the Knight doublet is shown [11] on the left, much weaker and not interfering with the coherence transfer doublet. Figure 5 is the first demonstration of the two effects together. 


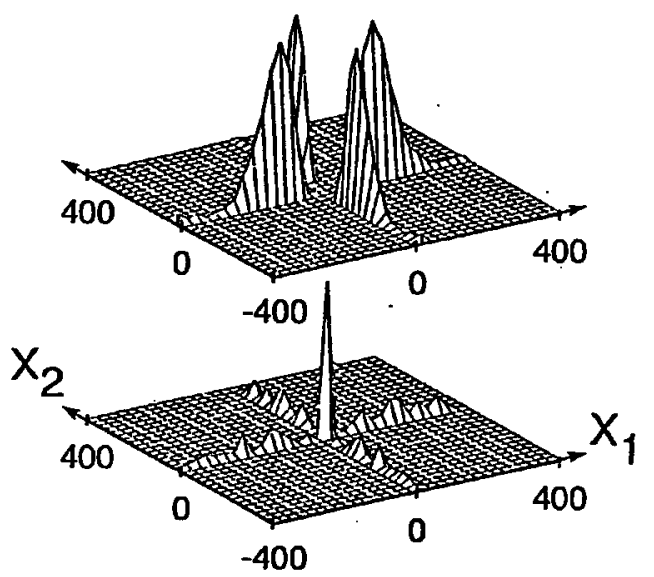

Fig. 3. The spatial probability distribution at $t=13$ cycles, for the two-clectron wave functions of Fig. 2. The upper distribution, corresponding to field strength $\mathcal{E}=0.01$ (cf. Fig. 2a), shows no probability at the origin. This means that the two-clectron ground state has been completely depleted. Also there is probability concentrated along the axes, indicating that one electron has been detached, but not both. In the lower distribution, for the higher field strength $\mathcal{E}=0.02$ a.u. (cf. Fig. 2b), some probability nevertheless remains at the origin, indicating that the two-clectron ground state remains partially occupied. Such a lower degree of detachment for a higher field strength is a signal of stabilization.

The recurring theme of e-e correlation makes it interesting to ask about every strong-field multi-electron process "how correlated is it?" Moreover, this raises the question whether a "degree of correlation" can be determined. If a degree of correlation could be formulated, this degrce could be calculated in principle for any wave function, and it could even be monitored as a function of time during an interaction. A time-dependent degree of correlation might even lead in some interaction processes to the recognition that correlation is subject to external control.

A specific definition of a degree of correlation which has simple and attractive properties has recently been proposed [12], and we will describe it here. The so-called "canonical representation" [13] of a two-electron atomic state $\Psi(x, y)$ is characterized by a (unique) single-sum basis expansion

$$
\Psi(x, y)=\sum_{\alpha} D_{\alpha} b_{\alpha}(x, y)
$$

where $b_{\alpha}(x, y)$ is given for electrons by a Slater determinant. We can first identify a degree of correlation $K$ roughly with the number of different functions $b_{\alpha}$ necessary to construct the exact two-particle wave function in its canonical representation. Of course, the number of non-zero $b_{\alpha}$ 's could easily be infinite, so to make a precise definition of $K$ from this rough idea, we have proceeded as follows. The normal- 


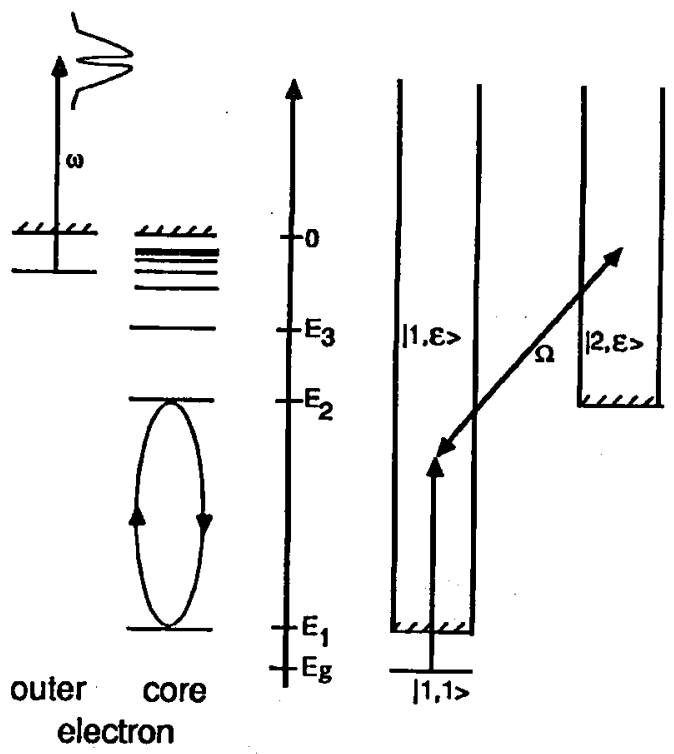

Fig. 4. On the left is shown an energy level diagram which pretends that one of the two electrons can be identified as "outer" and the other as "core". The sketch shows the two electrons behaving differently in the same laser pulse. The outer electron is being detached, with an unusual splitting in its photo-spectrum, and the inner electron is undergoing Rabi oscillations on its lowest transition. On the right a proper two-electron energy level diagram indicates the transitions that are responsible for the behavior sketched on the left.

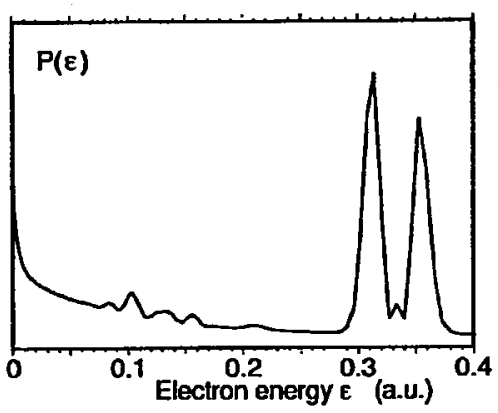

Fig. 5. Photo-electron spectrum created by a 40-cycle incident laser pulse (sine-squared envelope), with $\mathcal{E}=0.05$ a.u. and $\omega$ chosen to be resonant with the lowest core transition. The small multiple peak near to $\varepsilon=0.14$ a.u. is the Knight doublet $[10,11]$, while the large peak is the one due to coherence transfer.

ization for $\Psi(x, y)$ leads to the condition for the coeflicients $\sum_{\alpha}\left|D_{\alpha}\right|^{2}=1$, which shows that each squared coefficient can be interpreted as a weight (probability). The average of the probability $\left|D_{\alpha}\right|^{2}$ is then given by $\sum_{\alpha}\left|D_{\alpha}\right|^{2} \times\left|D_{\alpha}\right|^{2}$ and the 
inverse of this is the "number" of eflectively non-zero probabilities, so $K$ is then defined as

$$
K \equiv\left(\sum_{\alpha}\left|D_{\alpha}\right|^{4}\right)^{-1}
$$

Although $K$ has attractive properties, there is still a question how to compute it, even if we know the exact two-electron wave function $\Psi(x, y)$, because $\Psi$ will only rarely be expressed in Everett's canonical form. The key role in overcoming this practical problem is played by the single particle density operator $\rho\left(x, x^{\prime}\right)$ which is obtained from the total density matrix $\Psi \otimes \Psi^{*}$ by integrating out all particle coordinates and spins with the exception of those for one particle

$$
\rho\left(x, x^{\prime}\right) \equiv \int \mathrm{d} y \Psi(x, y) \Psi^{*}\left(x^{\prime}, y\right) .
$$

The desired coefficients $\left|D_{\alpha}\right|^{2}$ are precisely the eigenvalues of $\rho\left(x, x^{\prime}\right)$, so the practical problem reduces to the diagonalization of $\rho\left(x, x^{\prime}\right)$, which is numerically straightforward.

We have done this, and calculated $K$ as a function of time, for our two-electron model atom in a number of different situations, as is shown in Fig. 6. In each case the atom starts in its ground state. In case (a) a weak field induces

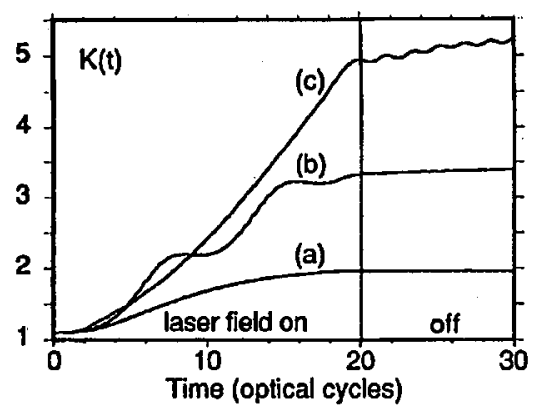

Fig. 6. The "degree of correlation" $K$ as a function of time, for our two-electron atom undergoing laser excitation with three different field strengths and frequencies. Curve (a) is appropriate to weak excitation and only little detachment, with $\mathcal{E}=0.005$ a.u. and $\omega=0.08$ a.u., curve (b) is for the resonant situation of Figs. 4 and 5 , with $\mathcal{E}=0.05$ a.u. and $\omega=0.395$ a.u., and curve (c) is for rapid two-electron detachment and ionization, with $\mathcal{E}=0.5$ a.u. and $\omega=1.0$ a.u.

photodetachment of the outer electron, with little or no involvement of the core electron. In cases (b) and (c) successively stronger fields are applied. Case (b) corresponds exactly to the situation already shown in Figs. 4 and 5, where a core resonance induces the Rabi oscillations while the outer electron is detached, and the Rabi oscillations can be seen now in the $K$ time dependence. Case (c) was calculated from a rapidly detaching outer electron, with strong involvement of the core electron as well. It is clear that the strength of the laser field has a pronounced influence on the degree of correlation, with stronger fields driving it 
to higher values. This is natural, and consistent with the picture that the number of final states available to a laser-excited pair of electrons can be large, so that a large number of orbitals may be required to describe the two-electron behavior.

It is interesting to see here that $K$ does not grow very rapidly, at least not much more rapidly than the corresponding detachment probabilities in the cases shown. Our data so far leave open the interesting question what interaction processes can cause $K$ to shrink instead of grow, although the stair-step character of curve (b) indicates that the natural growth of $K$ during detachment and ionization can be dynamically suppressed.

I am pleased to acknowledge conversations with my colleagucs Rainer Grobe and Kazimierz Rzążewski, who have been full collaborators in the development of the $K$ parameter. This research was partially supported by the US National Science Foundation under grants PHY92-00542 and INT89-05178.

\section{References}

[1] J.H. Eberly, Hyperfine Intcract. 37, 33 (1987).

[2] J. Javanainen, J.II. Eberly, Q. Su, Phys. Rev. A 38, 3430 (1988).

[3] J. Javanainen, J.IH. Eberly, Phys. Rev. A 39, R458 (1989).

[4] J.H. Eberly, Q. Su, J. Javanainen, Phys. Rev. Lett. 60, 861 (1989).

[5] Q. Su, J.II. Eberly, J. Javanainen, Phys. Rev. Lett. 62, 682 (1990).

[6] R. Grobe, J.H. Eberly, Phys. Rev. Lett. 68, 2905 (1992).

[7] R. Grobe, J.H. Eberly, Phys. Rev. A 47, R1605 (1993).

[8] R. Grobe, J.H. Eberly, in: Super Intense Laser Atom Physics, Eds. B. Piraux, A. L'Huillier, K. Rząziewski, Plenum, New York 1993, p. 445.

[9] R. Grobe, J.H. Eberly, Phys. Rev. A 48, 4664 (1993).

[10] P.L. Knight, Opt. Commun. 22, 172 (1977); J. Phys. B 11, L511 (1978).

[11] The Knight "doublet" in Fig. 4 has more than two maxima because of the coherence associated with the smooth laser pulse turn-on. This effect was described by K. Rzążewski, Phys. Rev. A 28, 2565 (1983), and D. Rogus, M. Lewenstein, J. Phys. $B$ 19, 3051 (1986).

[12] R. Grobe, K. Rzążewski, J.II. Eberly, submitted for publication.

[13] See H. Everet III, Rev. Mfod. Phys. 29, 454 (1957). 